\title{
Pre-Equalization for DS-UWB Systems with Spectral Mask Constraints
}

\author{
Amir-Hamed Mohsenian-Rad ${ }^{\dagger}$, Jan Mietzner ${ }^{\ddagger}$, Robert Schober ${ }^{\dagger}$, and Vincent W.S. Wong ${ }^{\dagger}$ \\ ${ }^{\dagger}$ Department of Electrical and Computer Engineering, University of British Columbia, Vancouver, Canada \\ $\ddagger$ EADS Defence \& Security, COM-EW Algorithms \& Software, Ulm, Germany \\ E-mail: \{hamed, rschober, vincentw\}@ece.ubc.ca, jan.mietzner@eads.com
}

\begin{abstract}
In this paper, we propose a novel optimizationbased pre-equalization filter (PEF) design for direct-sequence ultra-wideband (DS-UWB) systems with pre-Rake combining. The key feature in our design is that we explicitly take into account the spectral mask constraints that are usually imposed by the telecommunication regulation bodies. This avoids the need for an inefficient power back-off, which is necessary for transmit structures designed solely based on average transmit power constraints. Simulation results confirm that the proposed PEF design leads to significant performance gains over UWB PEF structures without explicit spectral mask considerations.
\end{abstract}

\section{INTRODUCTION}

Ultra-wideband (UWB) is an emerging technology for highrate short-range transmission. Due to their extremely large bandwidths, UWB systems can resolve even dense multipath components such that Rake combining can be used at the receiver to significantly reduce the negative impacts of fading in the received signal [1]. However, for many UWB applications, the receiver is a portable device with limited signal processing capabilities, making the implementation of Rake combiners with a sufficiently large number of fingers challenging.

To overcome this problem, a promising approach is to move computational complexity from the receiver to the more powerful transmitter (e.g., an access point). In this regard, pre-Rake combining can be used [2]-[4]. Pre-Rake combining exploits the reciprocity of the UWB radio channel, which was recently experimentally confirmed in [5]. Ideally, with preRake combining at the transmitter, channel estimation, diversity combining, and equalization are avoided at the receiver, and a simple symbol-by-symbol detector can be used [6].

However, pre-Rake combining has some serious drawbacks. In particular, for the long channel impulse responses (CIRs), which are typical for UWB applications, it may entail a relatively high error floor if simple symbol-by-symbol detection is applied at the receiver [2], [7]. To remedy this problem, while still keeping the receiver simple, pre-equalization can be used at the transmitter to effectively decrease the residual intersymbol interference (ISI) at the receiver [8]-[10].

Most of the previous work on pre-Rake and pre-equalizer design (e.g., in [2]-[10]) includes only constraints to limit the (average) transmit power. However, prior studies do not include constraints to limit the power spectral density (PSD) of the transmitted UWB signals. This can severely affect the overall system performance as most of the telecommunication regulation bodies, e.g., the US Federal Communications Commission (FCC), impose spectral mask constraints to limit
UWB emission levels in order to prevent the harmful interference on incumbent legacy narrow-band receivers. In such a setting, the existing UWB pre-filtering techniques can be far from optimal in practice, as they require an appropriate power back-off so that the spectral masks are not violated.

In this paper, we propose a novel pre-equalization filter (PEF) design for direct-sequence (DS) UWB systems [11]. We first formulate an elaborate optimization problem, with the coefficients of the PEF as the optimization variables, which includes constraints with respect to the spectral mask, the energy concentration in a single tap within the resulting overall CIR (in order to reduce residual ISI), and the overall transmit power. To the best of our knowledge, this work is the first to explicitly consider the spectral mask for pre-filter design in DS-UWB systems. We use a non-convex optimization technique, called semi-definite relaxation, to solve the formulated optimization problem to achieve a close-to-optimal PEF design. Our simulation results confirm that our proposed PEF scheme leads to significant performance gains over PEF structures without spectral mask considerations. Note that our work is different from the previous work on UWB pulseshaping with spectral masks, e.g., in [12]. The pre-equalization design problem and ISI reduction are not addressed in [12].

Organization: The system model and the PEF design are presented in Sections II and III, respectively. Simulation results are given in Section IV. The paper is concluded in Section V.

Notation: $\mathcal{E}\{\cdot\},[\cdot]^{T},(\cdot)^{*},[\cdot]^{H}, \Re\{\cdot\},\lceil\cdot\rceil, \delta(\cdot)$, and $*$ denote expectation, transposition, complex conjugation, Hermitian transposition, the real part of a complex number, the ceiling function, the Dirac delta function, and convolution, respectively. Also $X\left(e^{j \omega}\right) \triangleq \sum_{k=-\infty}^{\infty} x[k] e^{-j \omega k}, \Phi_{x x}\left(e^{j \omega}\right) \triangleq$ $\sum_{\tau=-\infty}^{\infty} \phi_{x x}[\tau] e^{-j \omega \tau}$, and $\phi_{x x}[\tau] \triangleq \mathcal{E}\left\{x[k] x^{*}[k-\tau]\right\}$ denote the discrete-time Fourier transform, the power spectral density, and the autocorrelation function of $x[k]$, respectively. Depending on the context, $x[k]$ represents either a sequence or the $k$ th element of a sequence. Finally, $X(j \Omega) \triangleq \int_{-\infty}^{+\infty} x(t) e^{-j \Omega t} d t$ denotes the continuous-time Fourier transform of $x(t)$.

\section{SySTEM MOdEL}

Consider a DS-UWB system [6] with a single transmit antenna and a single receive antenna. A block diagram of the discrete-time model of such a system is depicted in Fig. 1. We denote the symbol duration by $T_{s}$ and the chip duration by $T_{c}=T_{s} / N$, where $N$ is the spreading factor. All signals and systems are represented by their complex baseband equivalents. Next, we explain the details of the system model. 


\section{A. Transmitter Structure}

At the transmit antenna, a train of independent and identically distributed (i.i.d.) data symbols $a[n] \in\{ \pm 1\}$ is filtered with PEF $f[n]$ of length $L_{f}$, and the filter output signal is ${ }^{1}$

$$
v[n] \triangleq f[n] * a[n]=\sum_{l=0}^{L_{f}-1} f[l] a[n-l] .
$$

We will optimize the PEF for minimization of the amount of residual ISI at the receiver in Section III. The output signal of the PEF is up-sampled by a factor of $N \geq 1$ :

$$
\tilde{v}[k]= \begin{cases}v[n], & \text { if } k=N n, \\ 0, & \text { if } k \neq N n .\end{cases}
$$

It is then filtered with a (real-valued) spreading sequence $c[k]$, $0 \leq k<N$, which is normalized such that $\sum_{k=0}^{N-1}|c[k]|^{2}=1$. The resulting transmit sequence $s[k]$ is given by

$$
s[k]=\tilde{v}[k] * \tilde{g}[k]=\sum_{i=-\infty}^{\infty} v[i] \tilde{g}[k-i N],
$$

where $\tilde{g}[k] \triangleq c[k] * g[k]=\sum_{i=0}^{N-1} c[i] g[k-i]$ includes the combined effects of the pre-Rake filter $g[k]$ and spreading sequence $c[k]$. Here, we consider a general transmitter structure as we do not impose any restrictions on $c[k]$ and $g[k]$. If a spreading sequence is not applied, e.g. as in [2], [3], [5], we simply have $c[0]=1$ and $c[k]=0,1 \leq k<N$. In general, $g[k]$ depends on the CIR $h[k]$, which has length $L_{h}$. For example, in an all-pre-Rake (also called time-reversal) filter, we have [2]

$$
g[k] \triangleq h^{*}\left[L_{h}-k-1\right], \quad 0 \leq k<L_{g}, \quad L_{g}=L_{h} .
$$

\section{B. Channel Model}

The equivalent baseband discrete-time CIR

$$
\left.h[k] \triangleq g_{T}(t) * h(t) * g_{R}(t)\right|_{k T_{c}},
$$

contains the combined effects of the transmit filter $g_{T}(t)$, the continuous-time CIR $h(t)$, and the receive filter $g_{R}(t)$, sampled at chip interval $T_{c}{ }^{2}$ For the wireless channel, we adopt the IEEE 802.15.3a channel model [13], [14]. Consequently, the passband version $h^{\prime}(t)$ of the baseband CIR $h(t)$ consists of $L_{c}$ clusters of $L_{r}$ rays and is modeled as

$$
h^{\prime}(t)=\vartheta \sum_{l=1}^{L_{c}} \sum_{k=1}^{L_{r}} \rho_{k, l} \delta\left(t-T_{l}-\tau_{k, l}\right),
$$

where $T_{l}$ is the delay of the $l$ th cluster, $\tau_{k, l}$ is the delay of the $k$ th ray of the $l$ th cluster, $\rho_{k, l}$ is the random multipath gain coefficient, and $\vartheta$ models the lognormal shadowing. In [13], [14], four parameter sets for the various channel model (CM) parameters in (6) are specified. The resulting channel models are known as CM1, CM2, CM3, and CM4. They represent different usage scenarios and different amounts of ISI.

\footnotetext{
${ }^{1}$ Here, we place the PEF before the up-sampling, as in [8]. This helps to reduce the length of the required PEF. However, our design is also applicable to the case when the PEF is placed after the up-sampling, e.g., as in [9].

${ }^{2}$ Note that the continuous-time overall CIR $g_{T}(t) * h(t) * g_{R}(t)$ has infinite length. However, the discrete-time version $h[k]$ can be truncated to a finite length $L_{h}$ with negligible energy loss if $L_{h}$ is chosen sufficiently large.
}

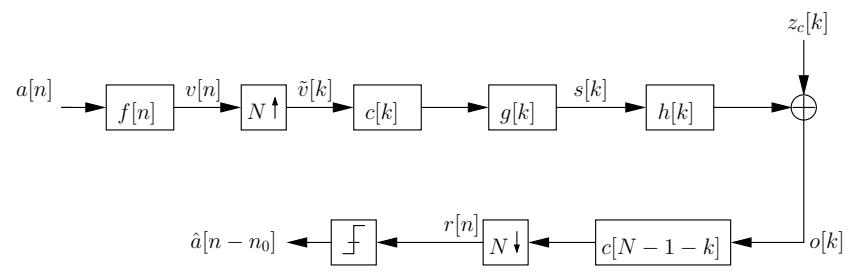

Fig. 1. Block diagram of DS-UWB system with one transmit antenna, pre-equalization, pre-Rake combining, and one receive antenna.

\section{Receiver Structure}

The received signal is obtained as

$$
o[k]=\sum_{l=0}^{L_{h}-1} h[l] s[k-l]+z_{c}[k],
$$

where $z_{c}[k]$ denotes the chip-level additive white Gaussian noise (AWGN) with variance $\sigma_{c}^{2} \triangleq \mathcal{E}\left\{\left|z_{c}[k]\right|^{2}\right\}$. Received signal $o[k]$ is filtered with the time-reversed spreading sequence $c[N-1-k], 0 \leq k<N$, and is down-sampled at times $k=N n+k_{0}$, where $0 \leq k_{0}<N$ denotes the sampling phase. The resulting receiver output signal $r[n]$ can be expressed as

$$
r[n]=\sum_{l=-\infty}^{\infty} q\left[N l+k_{0}\right] v[n-l]+z_{s}[n],
$$

with an overall CIR

$$
q[k]=\tilde{g}[k] * \tilde{h}[k]=\sum_{i=0}^{L_{g}+N-2} \tilde{g}[i] \tilde{h}[k-i],
$$

and symbol-level noise

$$
z_{s}[n]=\sum_{i=0}^{N-1} c[i] z_{c}\left[N(n-1)+k_{0}+i+1\right] .
$$

Here, $\tilde{h}[k]$ includes the combined effects of the channel filter $h[k]$ and the time-reversed spreading sequence $c[N-1-k]$ :

$$
\tilde{h}[k] \triangleq h[k] * c[N-1-k]=\sum_{i=0}^{N-1} c[i] h[k+i-(N-1)] .
$$

We notice that $z_{s}[n]$ is also an AWGN sample with variance $\sigma_{s}^{2} \triangleq \mathcal{E}\left\{\left|z_{s}[n]\right|^{2}\right\}=\sigma_{c}^{2} \sum_{i=0}^{N-1}|c[i]|^{2}=\sigma_{c}^{2}$.

Since our goal is to design a UWB system with minimal complexity at the receiver, no additional filtering is applied at the receiver, and symbol decisions are made according to

$$
\hat{a}\left[n-n_{0}\right]=\operatorname{sign}\{\Re\{r[n]\}\},
$$

where $\hat{a}\left[n-n_{0}\right]$ is the estimate for $a\left[n-n_{0}\right], n_{0}$ denotes the decision delay, and $\operatorname{sign}\{x\}=1$ if $x \geq 0$ and $\operatorname{sign}\{x\}=-1$ otherwise. Notice that no equalizer is used at the receiver.

\section{Spectral Masks}

The telecommunication regulation and standardization bodies, such as the FCC in the US, impose regulations which limit the permissable PSD for UWB transmissions to prevent interference to legacy narrow-band receivers. The FCC spectral mask for outdoor communications is shown in Fig. 2 [15]. 


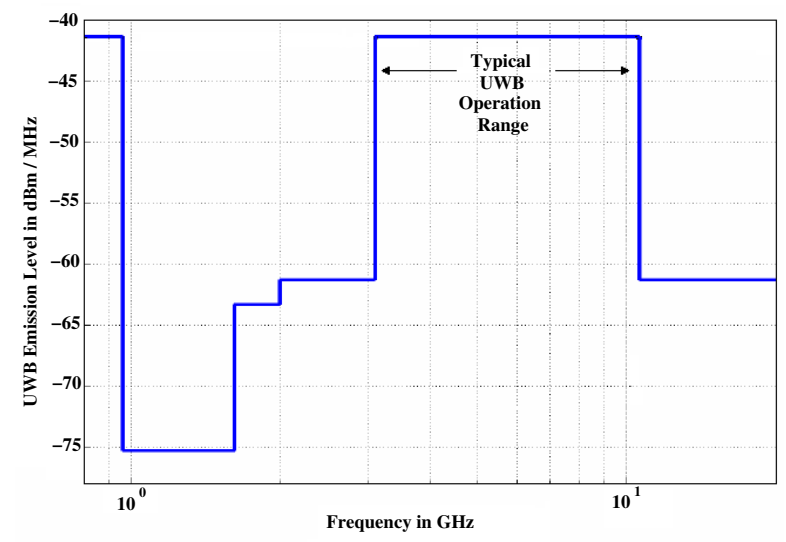

Fig. 2. FCC spectral mask for UWB transmissions in outdoor environment. A typical operation range for UWB systems is the $3.1-10.6 \mathrm{GHz}$ band.

We first notice that our system model is in discrete-time, while the spectral mask is usually defined in continuous-time. Correspondingly, let $\Omega$ and $\omega$ denote the angular frequency associated with the continuous-time and discrete-time Fourier transform, respectively. We define $\Omega_{\min }$ and $\Omega_{\max }$ as the minimum and maximum frequencies used by the UWB system (e.g., $\Omega_{\min }=2 \pi \times 3.5 \mathrm{GHz}$ and $\Omega_{\max }=2 \pi \times 4.5 \mathrm{GHz}$ [11]). Also let $m(\Omega)$ denote the spectral mask set by the FCC, etc. (e.g., $m(\Omega)=-41.3 \mathrm{dBm} / \mathrm{MHz}$ for any $\Omega_{\min } \leq \Omega \leq \Omega_{\max }$ ). We define $K=\frac{B_{s}}{1 \mathrm{MHz}}+1$, where $B_{s}=\Omega_{\max }-\Omega_{\min }$ denotes the total bandwidth used by the designed UWB system. Finally, let $\Omega_{1}, \ldots, \Omega_{K}$ denote the discrete frequency levels which uniformly spread out over the bandwidth $B_{s}$. Clearly, $\Delta \Omega=\Omega_{2}-\Omega_{1}=\ldots=\Omega_{K}-\Omega_{K-1}=2 \pi \times 1 \mathrm{MHz}$. For each $\mu=1, \ldots, K$, it is required that [15]

$$
\int_{\omega_{\mu}-\frac{\Delta \omega}{2}}^{\omega_{\mu}+\frac{\Delta \omega}{2}} \Phi_{s s}\left(e^{j \omega}\right) d \omega \leq \int_{\Omega_{\mu}-\frac{\Delta \Omega}{2}}^{\Omega_{\mu}+\frac{\Delta \Omega}{2}} m(\Omega) d \Omega
$$

where $\omega_{\mu} \triangleq T_{c} \Omega_{\mu}\left[16\right.$, Ch. 1.7], $\Delta \omega \triangleq T_{c} \Delta \Omega$, and $\Phi_{s s}\left(e^{j \omega}\right)$ denotes the power spectral density for transmitted signal $s[k]$. On the other hand, we can show that

$$
\Phi_{s s}\left(e^{j \omega}\right)=\left|\tilde{G}\left(e^{j \omega}\right)\right|^{2} \Phi_{\tilde{v} \tilde{v}}\left(e^{j \omega}\right)
$$

where $\tilde{G}\left(e^{j \omega}\right)=\mathcal{F}\{\tilde{g}[k]\}$. We also have

$$
\Phi_{\tilde{v} \tilde{v}}\left(e^{j \omega}\right)=\left|F\left(e^{j \omega N}\right)\right|^{2} \Phi_{a a}\left(e^{j \omega N}\right)=\left|F\left(e^{j \omega N}\right)\right|^{2},
$$

where $F\left(e^{j \omega N}\right)=\sum_{k=0}^{L_{f}-1} f[k] e^{-j \omega N k}$ and $\Phi_{a a}\left(e^{j \omega N}\right)=1$ due to the i.i.d. assumption for the data symbols $a[n]$. From (14) and (15), and assuming that the spectral mask $m(\Omega)$ and $\operatorname{PSD} \Phi_{s s}\left(e^{j \omega}\right)$ are almost constant over $\Omega_{\mu}-\frac{\Delta \Omega}{2} \leq \Omega \leq$ $\Omega_{\mu}+\frac{\Delta \Omega}{2}$ and $\omega_{\mu}-\frac{\Delta \omega}{2} \leq \omega \leq \omega_{\mu}+\frac{\Delta \omega}{2}$, respectively, for each $\mu=1, \ldots, K$, inequality (13) becomes

$$
\left|\tilde{G}\left(e^{j \omega_{\mu}}\right)\right|^{2}\left|F\left(e^{j \omega_{\mu} N}\right)\right|^{2} \leq M\left(\Omega_{\mu}\right) \triangleq \frac{m\left(\Omega_{\mu}\right)}{T_{c}} .
$$

We can enforce the above inequality by tuning the coefficients in the PEF, i.e., $f[0], \ldots, f\left[L_{f}-1\right]$, as we will discuss next.

\section{OPtIMIZATION-BASED PRE-EQUALIZATION With SPECTRAL MASK CONSTRAints}

In this section, we propose an optimization framework to design an efficient PEF with explicit spectral mask provisioning. We first develop an elaborate model for a non-convex optimization problem with respect to the coefficients of the PEF. We then solve the formulated problem by using semi-definite relaxation and semi-definite convex programming techniques.

\section{A. Problem Formulation}

It is convenient to first rewrite (8) as

$$
r[n]=(\mathbf{Q} \mathbf{f})^{H} \mathbf{a}[n]+z_{s}[n],
$$

where $\mathbf{a}[n] \triangleq\left[a[n] \ldots a\left[n-L_{t}+1\right]\right]^{T}, \mathbf{f} \triangleq\left[f[0] \ldots f\left[L_{f}-\right.\right.$ 1]] $]^{H}$, and $\mathbf{Q}$ is an $L_{t} \times L_{f}$ column-circulant matrix with vector

$$
\left[q\left[k_{0}\right] q\left[N+k_{0}\right] \ldots q\left[N\left(L_{q}-1\right)+k_{0}\right] \mathbf{0}_{L_{f}-1}^{T}\right]^{H}
$$

as its first column. Here,

$$
L_{t} \triangleq L_{q}+L_{f}-1
$$

and $L_{q}=\left\lceil\left(L_{g}+L_{h}+2 N-3\right) / N\right\rceil$ are the length of the impulse response of the overall system (including the PEF) and of the sampled overall CIR $q\left[N n+k_{0}\right]$, respectively. Next, we study PEF design including various design aspects.

1) Spectral Mask Constraints: We start by taking into account the spectral mask in (16). In vector form, we have

$$
\left|F\left(e^{j \omega N}\right)\right|^{2}=\mathbf{f}^{H} \mathbf{d}(N \omega) \mathbf{d}^{H}(N \omega) \mathbf{f},
$$

where

$$
\mathbf{d}(N \omega) \triangleq\left[\begin{array}{lll}
1 & e^{j \omega N T_{s}} & e^{j \omega 2 N T_{s}}
\end{array} \ldots e^{j \omega\left(L_{f}-1\right) N T_{s}}\right]^{T} .
$$

Therefore, the spectral mask in (16) imposes $K$ inequality constraints on the selection of the PEF coefficients $\mathbf{f}$ as

$\left|\tilde{G}\left(e^{j \omega_{\mu}}\right)\right|^{2} \mathbf{f}^{H} \mathbf{d}\left(N \omega_{\mu}\right) \mathbf{d}^{H}\left(N \omega_{\mu}\right) \mathbf{f} \leq M\left(\Omega_{\mu}\right), \quad \mu=1, \ldots, K$.

We notice that $\left|\tilde{G}\left(e^{j \omega_{\mu}}\right)\right|^{2}$ is fixed for each $\mu=1, \ldots, K$ as far as selecting the PEF coefficients $\mathbf{f}$ is concerned.

2) Energy Concentration: Since we assume that no equalizer is used at the receiver, it is required that for each received symbol, most of the channel energy is concentrated in a single channel tap. Considering (17), let $\mathbf{Q}_{\text {pre }}$ denote the submatrix of $\mathbf{Q}$ consisting of the first $\eta_{\text {pre }}$ rows. Also let $\mathbf{Q}_{\text {post }}$ denote the submatrix of $\mathbf{Q}$ consisting of the last $\eta_{\text {post }}$ rows. Here, $\eta_{\text {pre }}$ and $\eta_{\text {post }}$ are selected such that $\eta_{\text {pre }}+\eta_{\text {post }}+1=L_{t}$, where $L_{t}$ is as in (18). In this regard, we can rewrite $\mathbf{Q}$ as

$$
\mathbf{Q}=\left[\begin{array}{c}
\mathbf{Q}_{\text {pre }} \\
--- \\
\mathbf{Q}_{0} \\
--- \\
\mathbf{Q}_{\text {post }}
\end{array}\right] \text {. }
$$

Here, $\mathbf{Q}_{0}$ denotes a $1 \times L_{f}$ matrix which includes the $\left(\eta_{\text {pre }}+1\right)^{\text {th }}$ row of matrix $\mathbf{Q}$. We can rewrite (17) as

$$
\begin{aligned}
r[n]= & \left(\mathbf{Q}_{0} \mathbf{f}\right) a_{0}[n]+ \\
& \left(\mathbf{Q}_{\text {pre }} \mathbf{f}\right) \mathbf{a}_{\text {pre }}[n]+\left(\mathbf{Q}_{\text {post }} \mathbf{f}\right) \mathbf{a}_{\text {post }}[n]+z_{s}[n],
\end{aligned}
$$


where $a_{0} \triangleq a\left[n-n_{0}\right]=a\left[n-\eta_{\text {pre }}\right], \mathbf{a}_{\text {pre }} \triangleq[a[n] \ldots a[n-$ $\left.\left.\eta_{\text {pre }}-1\right]\right]^{T}$, and $\mathbf{a}_{\text {post }} \triangleq\left[a\left[n-\eta_{\text {pre }}+1\right] \ldots a\left[n-L_{t}+1\right]\right]^{T}$. We have to concentrate most energy of the overall CIR $\mathbf{Q} \mathbf{f}$ in a single high energy tap $\mathbf{Q}_{0} \mathbf{f}$, while keeping the residual ISI caused by the terms $\left(\mathbf{Q}_{p r e} \mathbf{f}\right) \mathbf{a}_{\text {pre }}[n]$ and $\left(\mathbf{Q}_{\text {post }} \mathbf{f}\right) \mathbf{a}_{\text {post }}[n]$ in (23) small in order to achieve a low bit error rate (BER). This introduces the following constraint on the PEF coefficients $\mathbf{f}$ :

$$
\mathbf{f}^{H} \mathbf{Q}_{p r e}^{H} \mathbf{Q}_{p r e} \mathbf{f}+\mathbf{f}^{H} \mathbf{Q}_{\text {post }}^{H} \mathbf{Q}_{\text {post }} \mathbf{f} \leq \alpha,
$$

where $\alpha$ is a design parameter which imposes an upper bound for the amount of residual ISI at the receiver. One possible choice that leads to a desirable system performance (as shown in Section IV) is to set $\alpha=\sigma_{s}^{2}$ in order to limit the ISI to be less than or equal to the noise variance.

We notice that our design goal on energy concentration in a single tap can also be interpreted in terms of the signal-tointerference-plus-noise-ratio (SINR) for each symbol:

$$
\operatorname{SINR}=\frac{\mathbf{f}^{H} \mathbf{Q}_{0}^{H} \mathbf{Q}_{0} \mathbf{f}}{\mathbf{f}^{H} \mathbf{Q}_{p r e}^{H} \mathbf{Q}_{p r e} \mathbf{f}+\mathbf{f}^{H} \mathbf{Q}_{\text {post }}^{H} \mathbf{Q}_{\text {post }} \mathbf{f}+\sigma_{s}^{2}} .
$$

Clearly, by maximizing $\mathbf{f}^{H} \mathbf{Q}_{0}^{H} \mathbf{Q}_{0} \mathbf{f}$, while suppressing $\mathbf{f}^{H} \mathbf{Q}_{\text {pre }}^{H} \mathbf{Q}_{\text {pre }} \mathbf{f}+\mathbf{f}^{H} \mathbf{Q}_{\text {post }}^{H} \mathbf{Q}_{\text {post }} \mathbf{f}$, we can increase the SINR, leading to better (i.e., lower) BER.

3) Power Constraint: Further to the PSD constraints, we may also want to limit the normalized average transmission power by including the following constraint [8], [9]:

$$
\mathcal{E}\left\{|s[k]|^{2}\right\}=\mathbf{f}^{H} \mathbf{\Phi} \mathbf{f} \leq 1 .
$$

Here, $\boldsymbol{\Phi}$ is a Hermitian Toeplitz matrix with vector

$$
\left[\phi[0] \phi[-N] \ldots \phi\left[-N\left(L_{f}-1\right)\right]\right]
$$

in its first row, where $\phi[k] \triangleq \tilde{g}[k] * \tilde{g}[-k]$, cf. [8, Appendix A].

Combining our considerations on spectral mask constraints, energy concentration, and the power constraint, the proposed PEF design is obtained as the optimal solution of the following optimization problem over complex-valued vector variable f:

$$
\begin{array}{cl}
\underset{\mathbf{f}}{\mathbf{m a x}} & \mathbf{f}^{H} \mathbf{Q}_{0}^{H} \mathbf{Q}_{0} \mathbf{f} \\
\text { s.t. } & \mathbf{f}^{H} \mathbf{Q}_{p r e}^{H} \mathbf{Q}_{p r e} \mathbf{f}+\mathbf{f}^{H} \mathbf{Q}_{\text {post }}^{H} \mathbf{Q}_{\text {post }} \mathbf{f} \leq \alpha \\
& \left|\tilde{G}\left(e^{j \omega_{1}}\right)\right|^{2} \mathbf{f}^{H} \mathbf{d}\left(N \omega_{1}\right) \mathbf{d}^{H}\left(N \omega_{1}\right) \mathbf{f} \leq M\left(\Omega_{1}\right) \\
& \vdots \\
& \left|\tilde{G}\left(e^{j \omega_{K}}\right)\right|^{2} \mathbf{f}^{H} \mathbf{d}\left(N \omega_{K}\right) \mathbf{d}^{H}\left(N \omega_{K}\right) \mathbf{f} \leq M\left(\Omega_{K}\right) \\
& \mathbf{f}^{H} \mathbf{\Phi} \mathbf{f} \leq 1 .
\end{array}
$$

We notice that problem (28) is a non-concave maximization problem as the objective function $\mathbf{f}^{H} \mathbf{Q}_{0}^{H} \mathbf{Q}_{0} \mathbf{f}$ is not concave ${ }^{3}$ with respect to $\mathrm{f}$. Thus, the standard gradient-based methods (cf. [17]) cannot be used for solving problem (28). Furthermore, problem (28) has many nonlinear constraints. Therefore, problem (28) can be difficult to solve. Nevertheless, we can find a close-to-optimal solution for optimization problem (28) using a semi-definite relaxation scheme, as we explain next.

\footnotetext{
${ }^{3}$ The objective function in maximization problem (28) is actually convex.
}

\section{B. Real-valued Representation}

Recall that vector $\mathbf{f}$ in (28) is complex-valued. Let $\mathbf{x}$ and $\mathbf{y}$ denote the real and imaginary parts of vector $\mathrm{f}$. We have

$$
\mathbf{f}=\mathbf{x}+j \mathbf{y} .
$$

By using simple calculus, we can obtain $2 L_{f} \times 2 L_{f}$ real-valued matrices $\boldsymbol{\Psi}_{0}, \boldsymbol{\Psi}_{\text {pre }}$, and $\boldsymbol{\Psi}_{\text {post }}$ such that

$$
\begin{gathered}
\mathbf{f}^{H} \mathbf{Q}_{0}^{H} \mathbf{Q}_{0} \mathbf{f}=\left[\begin{array}{ll}
\mathbf{x}^{T} & \mathbf{y}^{T}
\end{array}\right] \mathbf{\Psi}_{0}\left[\begin{array}{l}
\mathbf{x} \\
\mathbf{y}
\end{array}\right], \\
\mathbf{f}^{H} \mathbf{Q}_{\text {pre }}^{H} \mathbf{Q}_{\text {pre }} \mathbf{f}=\left[\begin{array}{ll}
\mathbf{x}^{T} & \mathbf{y}^{T}
\end{array}\right] \boldsymbol{\Psi}_{\text {pre }}\left[\begin{array}{l}
\mathbf{x} \\
\mathbf{y}
\end{array}\right], \\
\mathbf{f}^{H} \mathbf{Q}_{\text {post }}^{H} \mathbf{Q}_{\text {post }} \mathbf{f}=\left[\begin{array}{ll}
\mathbf{x}^{T} & \mathbf{y}^{T}
\end{array}\right] \mathbf{\Psi}_{\text {post }}\left[\begin{array}{l}
\mathbf{x} \\
\mathbf{y}
\end{array}\right] .
\end{gathered}
$$

Obtaining $\mathbf{\Psi}_{0}, \mathbf{\Psi}_{\text {pre }}$, and $\boldsymbol{\Psi}_{\text {post }}$ from $\mathbf{Q}_{0}, \mathbf{Q}_{\text {pre }}$, and $\mathbf{Q}_{\text {post }}$ is straightforward. In a similar way, and by using simple calculus, we can obtain $2 L_{f} \times 2 L_{f}$ real-valued matrix $\boldsymbol{\Lambda}_{\text {power }}$ such that

$$
\mathbf{f}^{H} \mathbf{\Phi} \mathbf{f}=\left[\begin{array}{ll}
\mathbf{x}^{T} & \mathbf{y}^{T}
\end{array}\right] \boldsymbol{\Lambda}_{\text {power }}\left[\begin{array}{l}
\mathbf{x} \\
\mathbf{y}
\end{array}\right] .
$$

Finally, we can obtain a $2 L_{f} \times 2 L_{f}$ real-valued matrix $\boldsymbol{\Gamma}\left(\omega_{\mu}\right)$, for each $\mu=1, \ldots, K$, such that

$$
\mathbf{f}^{H} \mathbf{d}\left(N \omega_{\mu}\right) \mathbf{d}^{H}\left(N \omega_{\mu}\right) \mathbf{f}=\left[\begin{array}{ll}
\mathbf{x}^{T} & \mathbf{y}^{T}
\end{array}\right] \boldsymbol{\Gamma}\left(\omega_{\mu}\right)\left[\begin{array}{l}
\mathbf{x} \\
\mathbf{y}
\end{array}\right] .
$$

For notational simplicity, we also define

$$
\gamma_{\mu} \triangleq \frac{M\left(\Omega_{\mu}\right)}{\left|\tilde{G}\left(e^{j \omega_{\mu}}\right)\right|^{2}}, \quad \mu=1, \ldots, K .
$$

We are now ready to rewrite problem (28) as the following optimization problem over real-valued vector variables:

$$
\begin{aligned}
& \underset{\mathbf{x}, \mathbf{y}}{\max }\left[\begin{array}{ll}
\mathbf{x}^{T} & \mathbf{y}^{T}
\end{array}\right] \boldsymbol{\Psi}_{0}\left[\begin{array}{l}
\mathbf{x} \\
\mathbf{y}
\end{array}\right] \\
& \text { s.t. } {\left[\begin{array}{ll}
\mathbf{x}^{T} & \mathbf{y}^{T}
\end{array}\right]\left(\boldsymbol{\Psi}_{\text {pre }}+\boldsymbol{\Psi}_{\text {post }}\right)\left[\begin{array}{l}
\mathbf{x} \\
\mathbf{y}
\end{array}\right] \leq \alpha, } \\
& {\left[\begin{array}{ll}
\mathbf{x}^{T} & \mathbf{y}^{T}
\end{array}\right] \boldsymbol{\Gamma}\left(\omega_{\mu}\right)\left[\begin{array}{l}
\mathbf{x} \\
\mathbf{y}
\end{array}\right] \leq \gamma_{\mu}, \quad \mu=1, \ldots, K, } \\
& {\left[\begin{array}{ll}
\mathbf{x}^{T} & \mathbf{y}^{T}
\end{array}\right] \boldsymbol{\Lambda}_{\text {power }}\left[\begin{array}{l}
\mathbf{x} \\
\mathbf{y}
\end{array}\right] \leq 1 . }
\end{aligned}
$$

We note that problems (28) and (36) are equivalent. In fact, their solutions can be converted into each other through the relationship in (29). Furthermore, we note that similar to problem (28), optimization problem (36) is non-concave.

\section{Semi-definite Relaxation}

We first introduce a new real-valued matrix $\mathbf{W}$ as

$$
\mathbf{W} \triangleq\left[\begin{array}{l}
\mathbf{x} \\
\mathbf{y}
\end{array}\right]\left[\begin{array}{ll}
\mathbf{x}^{T} & \mathbf{y}^{T}
\end{array}\right] .
$$

Clearly, matrix $\mathbf{W}$ is positive semi-definite (i.e., $\mathbf{W} \succeq \mathbf{0}$ ) and has unit rank. We also note that for any $2 L_{f} \times 2 L_{f}$ Hermitian matrix $A$, we have

$$
\left[\begin{array}{ll}
\mathbf{x}^{T} & \mathbf{y}^{T}
\end{array}\right] \mathbf{A}\left[\begin{array}{l}
\mathbf{x} \\
\mathbf{y}
\end{array}\right]=\operatorname{trace}(\mathbf{A W}) .
$$


Therefore, problem (36) is equivalent to

$$
\begin{array}{ll}
\mathbf{m a x}_{\mathbf{W} \succeq \mathbf{0}} & \operatorname{trace}\left(\boldsymbol{\Psi}_{0} \mathbf{W}\right) \\
\text { s.t. } & \operatorname{trace}\left(\left(\boldsymbol{\Psi}_{\text {pre }}+\mathbf{\Psi}_{\text {post }}\right) \mathbf{W}\right) \leq \alpha, \\
& \operatorname{trace}\left(\boldsymbol{\Gamma}\left(\omega_{\mu}\right) \mathbf{W}\right) \leq \gamma_{\mu}, \quad \mu=1, \ldots, K, \\
& \operatorname{trace}\left(\boldsymbol{\Lambda}_{\text {power }} \mathbf{W}\right) \leq 1, \\
& \operatorname{rank}(\mathbf{W})=1 .
\end{array}
$$

Problem (39) is still as difficult as problem (36), mainly due to the rank restriction constraint $\operatorname{rank}(\mathbf{W})=1$.

Next, we discard the rank restriction constraint and consider the following relaxed optimization problem:

$$
\begin{array}{ll}
\underset{\mathbf{W} \succeq \mathbf{0}}{\max } & \operatorname{trace}\left(\boldsymbol{\Psi}_{0} \mathbf{W}\right) \\
\text { s.t. } & \operatorname{trace}\left(\left(\boldsymbol{\Psi}_{\text {pre }}+\boldsymbol{\Psi}_{\text {post }}\right) \mathbf{W}\right) \leq \alpha, \\
& \operatorname{trace}\left(\boldsymbol{\Gamma}\left(\omega_{\mu}\right) \mathbf{W}\right) \leq \gamma_{\mu}, \quad \mu=1, \ldots, K, \\
& \operatorname{trace}\left(\boldsymbol{\Lambda}_{\text {power }} \mathbf{W}\right) \leq 1 .
\end{array}
$$

Problem (40) is a semi-definite programming (SDP) problem [18]. SDP is a generalization of linear programming (LP) over matrices (rather than vectors as in LP). Several solvers, such as SeDuMi [19] can efficiently solve the SDP problem in (40). Next, we explain how solving problem (40) can help us find close-to-optimal solutions for problem (39).

\section{PEF Design Algorithm}

Let $\mathbf{W}^{\star}$ denote the optimal solution for SDP problem (40). Clearly, if $\operatorname{rank}(\mathbf{W})=1$, then optimal solutions $\mathbf{x}^{\star}$ and $\mathbf{y}^{\star}$ for problem (39) can be obtained easily by using eigenvalue decomposition. However, in general, where $\operatorname{rank}(\mathbf{W})>1$, we can obtain close approximations of $\mathbf{x}^{\star}$ and $\mathbf{y}^{\star}$ by using a probabilistic method. In particular, we can use the following algorithmic steps which are based on the results in [20], [21]:

- Step 1. Using eigenvalue decomposition, obtain matrix $\mathbf{U}$ such that $\mathbf{W}^{\star}=\mathbf{U}^{\star} \mathbf{U}^{\star T}$ :

$$
\mathbf{W}^{\star}=\mathbf{V}^{\star T} \boldsymbol{\Sigma}^{\star} \mathbf{V}^{\star} \quad \Rightarrow \quad \mathbf{U}^{\star}=\mathbf{V}^{\star T} \boldsymbol{\Sigma}^{\star \frac{1}{2}},
$$

where $\mathbf{V}^{\star}$ is a unitary matrix and matrix $\boldsymbol{\Sigma}^{\star}$ is diagonal.

- Step 2. Using eigenvalue decomposition, obtain matrix $\Theta^{\star}$ such that $\boldsymbol{\Theta}^{\star T} \mathbf{U}^{\star T} \boldsymbol{\Psi}_{0} \mathbf{U}^{\star} \boldsymbol{\Theta}^{\star}$ becomes diagonal:

$$
\mathbf{U}^{\star T} \boldsymbol{\Psi}_{0} \mathbf{U}^{\star}=\boldsymbol{\Theta}^{\star} \boldsymbol{\Upsilon}^{\star} \boldsymbol{\Theta}^{\star T} \Rightarrow \boldsymbol{\Upsilon}^{\star}=\boldsymbol{\Theta}^{\star T} \mathbf{U}^{\star T} \boldsymbol{\Psi}_{0} \mathbf{U}^{\star} \boldsymbol{\Theta}^{\star},
$$

where $\mathbf{\Upsilon}^{\star}$ is a diagonal matrix.

- Step 3. Let $\zeta_{i}$, for each $i=1, \ldots, 2 L_{f}$, be i.i.d. random variables taking values -1 and 1 with equal probabilities. Also let $\boldsymbol{\zeta}=\left(\zeta_{1}, \ldots, \zeta_{2 L_{f}}\right)$. We select

$$
\left[\begin{array}{l}
\mathbf{x}^{\star} \\
\mathbf{y}^{\star}
\end{array}\right]=\frac{1}{\kappa_{\max }} \mathbf{U}^{\star} \boldsymbol{\Theta}^{\star} \boldsymbol{\zeta},
$$

where

$$
\begin{aligned}
& \kappa_{\max }=\max \left\{\max _{1 \leq \mu \leq K} \frac{\zeta^{T} \boldsymbol{\Theta}^{\star T} \mathbf{U}^{\star T} \boldsymbol{\Gamma}\left(\omega_{\mu}\right) \mathbf{U}^{\star} \boldsymbol{\Theta}^{\star} \zeta}{\gamma_{\mu}},\right. \\
& \zeta^{T} \boldsymbol{\Theta}^{\star T} \mathbf{U}^{\star T} \boldsymbol{\Lambda}_{\text {power }} \mathbf{U}^{\star} \boldsymbol{\Theta}^{\star} \zeta, \\
& \left.\frac{\zeta^{T} \boldsymbol{\Theta}^{\star T} \mathbf{U}^{\star T}\left(\boldsymbol{\Psi}_{\text {pre }}+\boldsymbol{\Psi}_{\text {post }}\right) \mathbf{U}^{\star} \boldsymbol{\Theta}^{\star} \zeta}{\alpha}\right\} .
\end{aligned}
$$

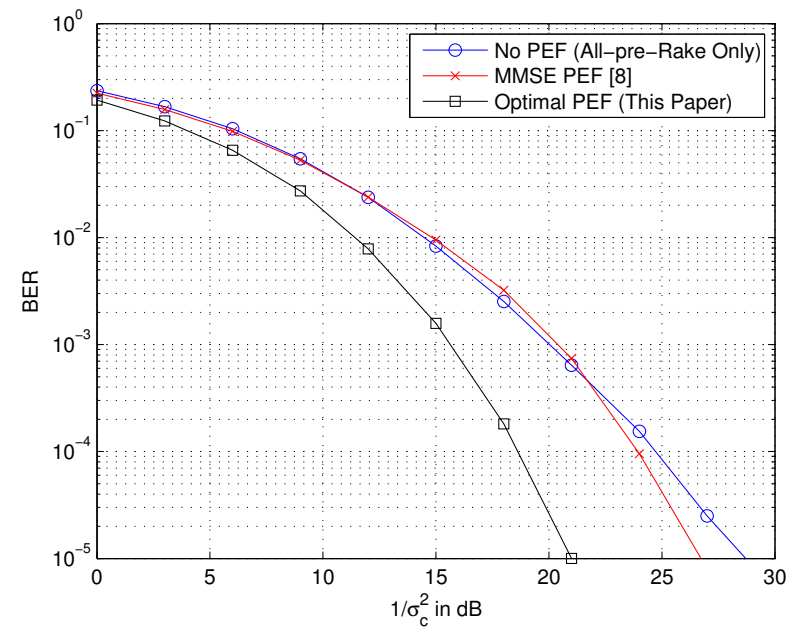

Fig. 3. BER vs. SNR $\frac{1}{\sigma^{2}}$ for all-pre-Rake filter [6] (with power back-off), MMSE PEF [8] (with power back-off), and our designed optimal PEF. All results are averaged over 500 channel realizations. For the MMSE PEF and also our designed optimal PEF, we set the filter length $L_{f}=10$.

We can verify that for any random choice of vector $\zeta$, the obtained $\mathrm{x}^{\star}$ and $\mathrm{y}^{\star}$ in (41) satisfy all the inequality constraints in problem (36). We then simply set

$$
\mathbf{f}^{\star}=\mathbf{x}^{\star}+j \mathbf{y}^{\star} \text {. }
$$

Let $\mathbf{f}_{o p t}$ denote the exact global optimal solution of PEF design optimization problem in (28). We notice that

$$
\mathbf{f}^{\star H} \mathbf{Q}_{0}^{H} \mathbf{Q}_{0} \mathbf{f}^{\star} \leq \mathbf{f}_{o p t}^{H} \mathbf{Q}_{0}^{H} \mathbf{Q}_{0} \mathbf{f}_{o p t} \leq \operatorname{trace}\left(\mathbf{\Psi}_{0} \mathbf{W}^{\star}\right),
$$

where the second inequality is due to the fact that problem (40) is less restrictive compared to problems (39), (36), and (28). From (43), the optimality loss in case of using our proposed algorithm in Section III-D is upper-bounded as

$$
\begin{aligned}
\frac{\mathbf{f}_{o p t}^{H} \mathbf{Q}_{0}^{H} \mathbf{Q}_{0} \mathbf{f}_{o p t}-\mathbf{f}^{\star H} \mathbf{Q}_{0}^{H} \mathbf{Q}_{0} \mathbf{f}^{\star}}{\mathbf{f}_{o p t}^{H} \mathbf{Q}_{0}^{H} \mathbf{Q}_{0} \mathbf{f}_{o p t}} & =1-\frac{\mathbf{f}^{\star H} \mathbf{Q}_{0}^{H} \mathbf{Q}_{0} \mathbf{f}^{\star}}{\mathbf{f}_{o p t}^{H} \mathbf{Q}_{0}^{H} \mathbf{Q}_{0} \mathbf{f}_{o p t}} \\
& \leq 1-\frac{\mathbf{f}^{\star H} \mathbf{Q}_{0}^{H} \mathbf{Q}_{0} \mathbf{f}^{\star}}{\operatorname{trace}\left(\mathbf{\Psi}_{0} \mathbf{W}^{\star}\right)} .
\end{aligned}
$$

By using the upper bound in (44), we have verified through simulations (not shown here) that the optimality loss for our design algorithm is small (less than $0.1 \%$ ) on average. Thus, a PEF design based on coefficients $\mathbf{f}^{\star}$ has almost the same performance as that achieved with the optimal coefficients $\mathbf{f}_{o p t}$. Moreover, by following the analysis in [22], we can show that the optimality loss is always guaranteed to be less than $36 \%$.

\section{Simulation Results}

In this section, we present simulation results for the proposed PEF design with pre-Rake combining and compare it with the all-pre-Rake combination (with no pre-equalization) in [6] and the minimum mean squared error (MMSE) preequalizer in [8], which also uses an all-pre-Rake.

Our simulation setting is as follows. The operational bandwidth $B_{s}=1 \mathrm{GHz}$ with $\Omega_{\min }=2 \pi \times 3.5 \mathrm{GHz}$ and $\Omega_{\max }=$ $2 \pi \times 4.5$ [11]. Therefore, $K=1001$. We also set the filter length $L_{f}=10$, the spreading factor $N=6$, parameters 


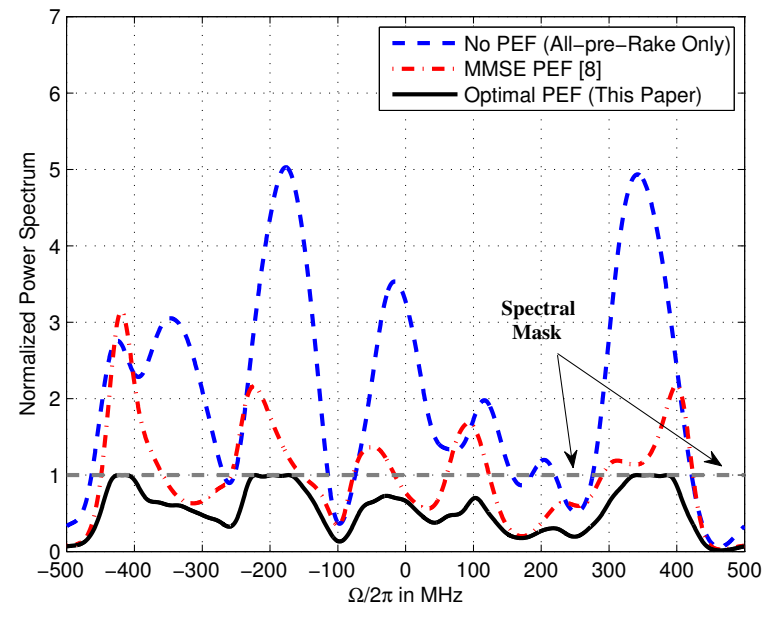

Fig. 4. Power spectrum in based-band of the transmitted signal within the $B_{s}=1 \mathrm{GHz}$ bandwidth for all-pre-Rake filter [2] (without power back-off), MMSE PEF [8] (without power back-off), and optimal PEF. Only the optimal PEF conforms the mandatory spectral mask. Both all-pre-Rake filter and the MMSE PEF require major power back-offs to be operational.

$\eta_{\text {pre }}=\eta_{\text {post }}=\left\lfloor\frac{L_{t}}{2}\right\rfloor$, and $\alpha=\sigma_{s}^{2}$. Here, we only include simulation results for the channel model CM1, where the parameters are according to [13]. The results for CM2, CM3, and CM4 are similar and omitted for brevity.

The simulation results for the achieved BER are shown in Fig. 3. We can see that our designed optimal PEF significantly improves the BER compared to the case that no PEF is included at the transmitter as in [3]-[7], and also the case when the MMSE PEF design without any spectral mask consideration is used as in [8]. The performance is poor for the MMSE PEF as the designed filter requires major power back-off to conform the mandatory spectral mask before being operational. On the other hand, the performance for the case with no PEF is even worse due to not only the impact of power back-off, but also the negative impact of the residual ISI since the receiver does not include an equalizer.

The spectral mask for the transmitted signal $s[k]$ is shown for a random channel realization in Fig. 4, where we normalized the spectral mask level to one. We can see that our designed PEF leads to a transmitted signal power spectrum that fully obeys the spectrum mask. In multiple frequency ranges (e.g., within 320 to $400 \mathrm{MHz}$ in the baseband) the power spectral density of the optimal PEF lies on the unit-level line, taking the shape of the imposed spectral mask. On the other hand, the all-pre-Rake combining as well as the MMSE PEF significantly violate the spectral mask within more than half of the operational frequency bandwidth. In fact, we can see that the power back-off required for the no PEF and the MMSE PEF cases in Fig. 4 are in factors of 3 and 5, respectively.

Last but not least, we note that the performance of the designed PEF can further be improved if we optimize parameter $\alpha$ in (24). However, this will be at the cost of extra computational complexity as in that case problem (40) would no longer be convex. Therefore, a fixed $\alpha$ might be a more appropriate choice in practice. As we saw in Figs. 3 and 4, the choice of $\alpha=\sigma_{s}^{2}$ already leads to an excellent performance.

\section{CONCLUSIONS}

We proposed a novel optimization-based PEF design for DS-UWB systems. Unlike previous work on pre-equalizer and pre-Rake design, here we explicitly took into account the spectral mask constraints which are usually imposed by regulation bodies. This avoids the need for an inefficient power back-off, which is necessary for the pre-filter designs in the existing literature. Simulation results confirmed that our proposed PEF design leads to significant performance gains over PEF structures without spectral mask considerations.

\section{REFERENCES}

[1] M. Win and R. Scholtz, "Characterization of Ultra-Wide Bandwidth Wireless Indoor Channels: A Communication-Theoretic View," IEEE J. Select. Areas Commun., vol. 20, pp. 1613-1627, Dec. 2002.

[2] T. Strohmer, M. Emami, J. Hansen, G. Papanicolaou, and A. Paulraj, "Application of Time-Reversal with MMSE Equalizer to UWB Communications," in Proc. of IEEE Globecom, Dallas, TX, Nov. 2004.

[3] H. Nguyen, I. Kovacs, and P. Eggers, "A Time Reversal Transmission Approach for Multiuser UWB Communications," IEEE Trans. Antennas and Propagation, vol. 54, pp. 3216-3224, Nov. 2006.

[4] Y.-H. Chang, S.-H. Tsai, X. Yu, and C.-C. Kuo, "Ultrawideband Transceiver Design Using Channel Phase Precoding," IEEE Trans. Signal Processing, vol. 55, pp. 3807-3822, July 2007.

[5] R. Qiu, C. Zhou, N. Guo, and J. Zhang, "Time Reversal With MISO for Ultrawideband Communications: Experimental Results," IEEE Antennas and Wireless Propagation Letters, vol. 5, pp. 269-273, Dec. 2006.

[6] W. Cao, A. Nallanathan, and C. Chai, "On the Tradeoff between Data Rate and BER Performance of Pre-RAKE DS UWB System," in Proc. of IEEE Globecom, San Francisco, CA, Nov. 2006.

[7] K. Usuda, H. Zhang, and M. Nakagawa, "Pre-Rake Performance for Pulse Based UWB System in a Standardized UWB Short-Range Channel," in Proc. of IEEE WCNC, Atlanta, GA, Mar. 2004.

[8] E. Torabi, J. Mietzner, and R. Schober, "Pre-Equalization for MISO DS-UWB Systems with Pre-Rake Combining," IEEE Trans. Wireless Commun., vol. 8, pp. 1295-1307, Mar. 2009.

[9] M. Emami, M. Vu, J. Hansen, A. Paulraj, and G. Papanicolaou, "Matched Filtering with Rate Back-off for Low Complexity Communications in Very Large Delay Spread Channels," in Proc. of the 38th Asilomar Conf. Signals, Systems, and Computers, Nov. 2004.

[10] X. Liao, S. Zhu, and E. Zeng, "Adaptive Interference Avoidance with Pre-RAKE Diversity Combining for High-Data-Rate UWB Systems," IEICE Trans. on Commun., vol. E91-B, no. 10, pp. 3347-3350, 2008.

[11] R. Fisher, R. Kohno, M. McLaughlin, and M. Welbourn, "DS-UWB Physical Layer Submission to IEEE 802.15 Task Group 3a (Doc. Number P802.15-03/0137r4)," Jan. 2005.

[12] X. Wu, Z. Tian, T. N. Davidson, and G. B. Giannakis, "Optimal Waveform Design for UWB Radios," IEEE Trans. Signal Processing, vol. 54, no. 6, pp. 2009-2021, June 2006.

[13] Channel Modeling Sub-Committee Final Report, "IEEE 802.1502/368r5-SG3a, IEEE P802.15,” Dec. 2002.

[14] A. Molisch, J. Foerster, and M. Pendergrass, "Channel Models for U1trawideband Personal Area Networks," IEEE Wireless Communications, vol. 10, pp. 14-21, Dec. 2003.

[15] "FCC Notice of Proposed Rule Making, Revision of Part 15 of the Commission's Rules Regarding Ultrawideband Transmission Systems," Communications Commission ET-Docket 98-153, May 2003.

[16] A. Oppenheim and R. Schafer, Digital Signal Processing. Englewood Cliffs, New Jersey: Prentice-Hall, Inc., 1975.

[17] S. Boyd and L. Vandenberghe, Convex Optimization. Cambridge University Press, 2004.

[18] L. Vandenberghe and S. Boyd, "Semidefinite Programming," SIAM Review, vol. 38, pp. 49-95, Mar. 1996.

[19] I. Polik, "SeDuMi User Guide," http://sedumi.ie.lehigh.edu, June 2005.

[20] S. He, Z. Q. Luo, J. Nie, and S. Zhang, "Semidefinite Relaxation Bounds for Indefinite Homogeneous Quadratic Optimization," SIAM Journal on Optimization, vol. 19, pp. 503-523, June 2008.

[21] A. Nemirovski, C. Roos, and T. Terlaky, "On Optimization of Quadratic Form over Intersection of Ellipsoids with Common Center,' Mathematical Programming, vol. 86, pp. 463-473, June 1999.

[22] Y. Nesterov, "Semidefinite Relaxation and Non-convex Quadratic Optimization," Optimization Methods and Software, vol. 12, pp. 1-20, 1997. 